P.A. NICKEL

KODAI MATH. J.

3 (1980), 59-69

\title{
A BIHARMONIC NORMAL OPERATOR
}

\author{
By PAUl A. NICKEL
}

ABSTRACT*. When a biharmonic singularity $s(x)$ is given on a boundary neighborhood $A$ of a Riemannian manifold $R$, there arises a rather natural question about the biharmonic extendability of this singularity to $p(x)$ which is biharmonic on all of $R$. For harmonic singularities $s(x) \in H(A)$, the question was answered by L. Sario (1952), who showed that although $s(x)$ may not be harmonically extendable, nevertheless, in terms of the regular singularity $L(f), s+L(f)$ is so extendable. Here, $L: \mathrm{C}(\partial A) \rightarrow H(A)$ is a bounded linear operator resembling the Dirichlet operator and is called normal. Analogously, in terms of $H^{2}(A)$, the set of biharmonic singularities on $A$, a biharmonic normal operator $L: \mathrm{C}(\partial A) \times \mathrm{C}(\partial A) \rightarrow H^{2}(A)$ is to resemble a Dirichlet operator and as an operator, is to be bounded. The purpose of the present effort is then to establish that, given a biharmonic normal operator $L$, each biharmonic singularity $s(x)$ has a biharmonic extension modulo a regular biharmonic function $L(f, g)$.

Examples may be obtained by applying the extension process to particular choices of $L$ and $s(x)$; in particular, when $s(x)$ has a fundamental biharmonic singularity at $a$, then a biharmonic Green's function with singularity at $a$ is obtained.

In his basic paper [4], L. Sario introduced a normal operator whose purpose was the construction of harmonic functions with certain prescribed behavior near the ideal boundary of a Riemann surface $W$. A full account of the applications of this operator, as well as an account of its own intrinsic interest, are given in, among others, the monograph of Rodin and Sario [1]. The issue of primary concern is, how does one accomplish showing that given a harmonic singularity $s(z)$ defined on a boundary neighborhood $W^{\prime} \subset W$, there is a harmonic $p(z)$ defined on a Riemann surface $W$ for which $p(z)-s(z)$ is a regular singularity $L(f)$. Here, in terms of $\alpha$, the compact border of the bordered $W^{\prime}, L$ is a continuous linear mapping similar to a Dirichlet operator. Explicitly, $L$ is a linear mapping from $\mathrm{C}(\alpha)$ to the set of regular singularities $H_{B}\left(W^{\prime}\right)$; that is, the set of bounded harmonic functions with 0 flux. In this sense, $p(z)$ is said

\footnotetext{
Received January 22, 1978.

* MOS Clessification $31 \mathrm{~B} 30$

Presented to the AMS at the New York City meeting, March 30, 1679.
} 
to have the same behavior as $s(z)$ at the boundary. For a given operator $L$ and singularity function $s(z)$, establishing the existence of $p \in H(W)$ for which $p(z)-s(z)$ is a regular singularity in $W^{\prime}$ is generally called the "principal function problem."

When the carrier of the harmonic functions and singularities is replaced by an $N$-dimensional Riemannian manifold $R$ with boundary neighborhood $A$, the so-called principle function problem may again be posed, and, such has been resolved in Sario, Schiffer, Glasner [6].

The analogous issue for biharmons functions is rather evident; that is, given a biharmonic singularity $s(x)$ defined on a boundary neighborhood $A \subset R$, can one show that $s(x)$ has a biharmonic continuation to $R$, modulo a regular biharmonic function $L(f, g)$, where $L$ is to be a linear operator resembling an operator which solves a biharmonic Dirichlet problem. The purpose here is to present the initial steps in the development of such a biharmonic normal operator. The setting of this presentation is to be a Riemannian manifold $R$ and the notation is to be consistent with that of Sario, et al [5]. So the locally compact, noncompact boundary neighborhood $A$ bordered by $\alpha$ is taken as $R-\bar{\Omega}_{\alpha}$, where $\bar{\Omega}_{\alpha}$ is a regular subregion; that is, the harmonic Dirichlet problem is solvable in $\Omega_{\alpha}$. In particular, a function $h(x)$ is called harmonic when $\Delta h=0$, where $\Delta$ is the usual Laplace-Beltrami operator. Moreover, the biharmonic Dirichlet problem is solvable in a regular region $\Omega$ as well; that is, in such a region there exists a biharmonic function $u(x)$ with prescribed values and Laplacian on the boundary $\partial \Omega$. And finally, flux integrals are $\int_{\beta} * d u$, where $d$ is the dimension raising differential operator, and $*$ is the Hodge operator carrying $p$-forms to $N-p$ forms. As usual, the orientation on $\beta=\partial D$ is that induced by $D$.

A careful examination of the original work of L. Sario [4], reveals that a good deal of the machinery presented there can, and will with appropriate interpretation, have meaning as well as use in the biharmonic case. Of course there are important differences. For example, in harmonic theory, if restrictions of harmonic functions to $A$ are to be singularity functions, then of course $\int_{\alpha} * d s=0$ is required of each singularity $s(x)$. On the other hand, $\int_{\alpha} * d u$ need not be 0 for biharmonic functions $u$ on $R$. But, in terms of $v_{\alpha}(x)$, quasiharmonic on $\Omega_{\alpha}$ and vanishing on $\alpha=\partial \Omega_{\alpha}$, it follows from the biharmonic Green's formula

$$
\int_{\alpha} u * d \Delta v-\Delta v * d u-v * d \Delta u+\Delta u * d v=0
$$

that $\int_{\alpha} * d u=\int_{\alpha} \Delta u * d v_{\alpha}$. Hence this flux condition is included in the defining requirements on biharmonic singularities as well as on this candidate for a normal biharmonic operator. With the analysis to follow is establshed the 
existence of such an operator that might reasonably be called normal, all in the spirit of the harmonic normal operator that has gone before it [4].

1. In terms of the boundary neighborhood $A=R-\bar{\Omega}_{\alpha}$, with smooth compact boundary $\alpha$, and $v_{\alpha}$, quasiharmonic on $\Omega_{\alpha}$ and vanishing on $\partial \Omega_{\alpha}=\alpha=\partial A$, a biharmonic normal operator $L$ a linear transformation from $\mathrm{C}(\alpha) \times \mathrm{C}(\alpha)$ into $H^{2}(A) \cap \mathrm{C}^{2}(\bar{A})$ which satisfies :

(i) $\left.L(f, g)\right|_{\alpha}=f \quad$ and $\left.\Delta L(f, g)\right|_{\alpha}=g$,

(ii) $\int_{\alpha} * d L(f, g)=\int_{\alpha} \Delta L * d v_{\alpha}$ and $\int_{\alpha} * d \Delta L(f, g)=0$,

(iii) $\operatorname{Sup}_{A}|L(f, g)| \leqq\|f\|+\lambda\|g\|$

for some $\lambda$ depending only on $L$, and $\operatorname{Sup}_{A}|\Delta L(f, g)| \leqq\|g\|$.

(iv) $L(f, 0)$ is harmonic on $A$ and satisfies $L(1,0)=1$.

In terms of the projection operator $P_{\alpha}: H^{2}(A) \cap \mathrm{C}^{2}(\bar{A}) \rightarrow \mathrm{C}(\alpha) \times \mathrm{C}(\alpha)$, with $P_{\alpha}(u)=$ $\left(\left.u\right|_{\alpha},\left.\Delta u\right|_{\alpha}\right)$, the defining property (i) may be written as $P_{\alpha} \circ L(f, g)=(f, g)$.

A function $s(x)$ belonging to $H^{2}(A) \cap \mathrm{C}^{2}(\dot{\bar{A}})$ is called a biharmonic singularity if $\int_{\alpha} * d s=\int_{\alpha} \Delta s * d v_{\alpha}$ and $\int_{\alpha} * d \Delta s=0$. The function $p(x)$ biharmonic on $R$ is said to have the singular behavior of $s(x)$ with respect to $L$ if

$$
p-s=L \circ P_{\alpha}(p-s)
$$

is valid in $A$; that is $p-s$ is a regular singularity because $p-s=L(f, g)$. On the other hand, when $p-s$ is a regular singularity then of course $L \circ P_{\alpha}(p-s)$ $=L \circ P_{\alpha} \circ L(f, g)=L(f, g)=p-s$ is valid in $A$.

2. The Main Theorem. Suppose that $L$ is a biharmonic normal operator defined with respect to the boundary neighborhood $A$ of the Riemannian manifold $R$, and that $s(x)$ is a biharmonic singularity on $A$. Then there exists $p$, biharmonic on $R$ such that $p(x)-s(x)$ is a regular biharmonic singularity for $L$. Furthermore, such a $p(x)$ is unique among those for which $p\left(x_{0}\right)=\Delta p\left(x_{0}\right)=0$.

The proof, presented in numbers $3,4,5$, and 6 , consists of solving an operator equation equivalent to (2) in a manner reminiscent of, but significantly different from, the harmonic case [1], [2].

3. The Operator Equation. Let $\Omega \supset \bar{\Omega}_{\alpha}$ be a regular region, and denote by $D(f, g)$ the solution in $\Omega$ to the biharmonic Dirichlet problem with boundary values given by $P_{\partial \Omega} \circ D(f, g)=(f, g)$. If for $T: \mathrm{C}(\partial \Omega) \times \mathrm{C}(\partial \Omega) \rightarrow \mathrm{C}(\partial \Omega) \times \mathrm{C}(\partial \Omega)$, given by $T(\phi, \phi)=P_{\hat{\alpha} \Omega} \circ L \circ P_{\alpha} \circ D(\phi, \phi)$, the functional equation

$$
(I-T)(\phi, \phi)=P_{\partial \Omega}\left(s-L \circ P_{\alpha}(s)\right)
$$


has a solution, then the problem of finding a biharmonic $p(x)$ which has the boundary behavior of $s(x)$ is solved by taking

$$
p(x)=\left\{\begin{array}{l}
L \circ P_{\alpha} \circ D(\phi, \phi)+s-L \circ P_{\alpha}(s) \text { on } A \\
D(\phi, \phi) \text { on } \Omega .
\end{array}\right.
$$

Of course, such a functional equation does have a solution if $T$ is completely continuous and has kernal 0 in an appropriately selected Banach space [7]. Furthermore, it now follows that such a $p(x)$ is well defined because on $A \cap \Omega$, the biharmonic functions $D(\phi, \phi)$ and $L \circ P_{\alpha} \circ D(\phi, \phi)+s-L \circ P_{\alpha}(s)$ agree since their values and Laplacians themselves agree on the boundary $\alpha \cup D \Omega$.

4. The Complete Continuity of $\mathbf{T}$. In the space $B=\mathrm{C}(\partial \Omega) \times \mathrm{C}(\partial \Omega)$, the norm $\|(f, g)\|$ is taken as the usual sum of sup norms $\|f\|+\|g\|$. And now the inequalities

$$
\begin{aligned}
& \sup _{\alpha}|D(f, g)|=\|D(f, g)\|_{\alpha} \leqq\|f\|+\mu\|g\|, \\
& \sup _{\alpha}|\Delta D(f, g)|=\|\Delta D(f, g)\|_{\alpha} \leqq\|g\|
\end{aligned}
$$

are established as preparation for showing that $T$ is completely continuous on the Banach space $B$.

Of course (5) is valid, since $\Delta D(f, g)$ is continuously equal to $g$ on $\partial \Omega$. To verify that (4) is valid, we represent $D(f, g)$ in terms of its Laplacian $H(x)$ and the harmonic Green's function $g_{\Omega}(x, y)$, as

$$
D(f, g)(x)=\int_{\Omega} g_{\Omega}(x, y) * H(y)+h(x)
$$

where $h(x)$ is harmonic on $\Omega$ with values $f$ on $\partial \Omega$. In fact, the integral term $I(x)$ is equal to 0 on $\partial \Omega$ and has Laplacian equal to $H(x)=g(x)$ there. But $g_{\Omega}(x, y)$ is of constant negative sign with flux +1 and of course the subharmonic $\int_{\Omega} g_{\Omega}(x, y) * 1_{y}=G l(x)$ attains its minimum $-\mu_{\Omega}$ at an interior point of $\Omega$. Hence the integral part of the expression for $D(f, g)$ is estimated as $|I(x)| \leqq \mu_{\Omega} \sup _{y \in \Omega}$ $|H(y)|=\mu_{\Omega} \max _{y \in \partial_{\Omega}}|g(y)|$. Of course $\sup _{x \in \Omega}|(h(x))|=\|f\|$, and the estimate (4) follows.

The boundedness of $T$ now follows easily from the estimates (4) and (5) along with the defining (iii) of $L$. That is, since $\partial \Omega \subset A$ and $\alpha \subset \Omega$, we write

$$
\begin{aligned}
\|T(f, g)\| & =\left\|P_{\partial \Omega} \circ L \circ P_{\alpha} \circ D(f, g)\right\|=\left\|\left.L \circ P_{\alpha} \circ D(f, g)\right|_{\partial \Omega}\right\|+\left\|\left.\Delta L \circ P_{\alpha} \circ D(f, g)\right|_{\partial \Omega}\right\| \\
& \leqq \\
& \leqq \\
& \leqq f(f, g)\left\|_{\alpha}+\lambda\right\| \Delta D(f, g)\left\|_{\alpha}+\right\| \Delta D(f, g) \|_{\alpha} \\
& \leqq\left(1+\mu_{\Omega}+\lambda\right)\|+\lambda\| g\|+\| g \| \\
&
\end{aligned}
$$


from which it follows that $T$ is bounded.

Next suppose that $\left(f_{n}, g_{n}\right)$ is a bounded sequence on $\mathrm{C}(\partial \Omega) \times \mathrm{C}(\partial \Omega)$. Then, of course $h_{n}=\Delta L \circ P_{\alpha} \circ D\left(f_{n}, g_{n}\right)$ is, with the estimate (5) and the defining (iii), a bounded sequence of harmonic functions on $A$, and has, as usual, a subsequence, say $\left(h_{n}\right)$ again, which converges uniformly to the harmonic $h(x)$ on compact sets of $A$. Of course $\partial \Omega$ is such a compact set, and so $\left.h_{n}\right|_{\partial \Omega}$, the second component of $T\left(f_{n}, g_{n}\right)$, converges in norm. In terms of the biharmonic $u_{n}=L \circ P_{\alpha} \circ D\left(f_{n}, g_{n}\right)$, the first component is then $\left.u_{n}\right|_{\partial \Omega}$.

We now require a regular region $\Omega^{\prime}$ slightly larger than $\Omega$, as well as a boundary neighborhood $A^{\prime}$ slightly smaller than $A$. So we choose $\Omega_{\alpha}^{\prime}$ and $\Omega^{\prime}$ for which $\bar{\Omega}_{\alpha} \subset \Omega_{\alpha}^{\prime} \subset \bar{\Omega}_{\alpha}^{\prime} \subset \Omega \subset \bar{\Omega} \subset \Omega^{\prime}$, and set $A^{\prime}$, with boundary $\alpha^{\prime}$ equal to $R-\bar{\Omega}_{\alpha}^{\prime}$. Of course $\partial \Omega \subset A^{\prime} \cap \Omega^{\prime}$.

In these terms, the boundary of $\Omega^{\prime} \cap A^{\prime}$ is $\alpha^{\prime} \cup \partial \Omega^{\prime}$, and its Green's function is $g_{\Omega^{\prime} \cap A^{\prime}}(x, y)$. Since $h_{n}(x) \rightarrow h(x)$ uniformly on compact sets of $A$, it follows that $w_{n}(x)=\int_{\Omega^{\prime} \cap A^{\prime}} g_{\Omega^{\prime} \cap A^{\prime}}(x, y) * h_{n}(y) \quad$ converges uniformly to $w(x)=$ $\int_{\Omega^{\prime} \cap A} g_{\Omega^{\prime} \cap A^{\prime}}(x, y) * h(y)$. In fact, $\Delta w_{n}(x) \rightarrow \Delta w(x)$, since $\Delta w_{n}=h_{n}$ and $\Delta w=h$. Now $w(x)$ is biharmonic and vanishes on $\alpha^{\prime} \cup \partial \Omega^{\prime}$. Hence, in terms of the harmonic $v_{n}(x)$, with $\left.v_{n}\right|_{\alpha^{\prime} \cup \partial \Omega^{\prime}}=\left.u_{n}\right|_{\alpha^{\prime} \cup \partial \Omega^{\prime}}$, it follows that $u_{n}(x)=w_{n}(x)+v_{n}(x)$. However,

$$
\left.v_{n}\right|_{\alpha^{\prime} \cup \partial \Omega^{\prime}}=\left.L \circ P_{\alpha} \circ D\left(f_{n}, g_{n}\right)\right|_{\alpha^{\prime} \cup \partial \Omega^{\prime}},
$$

so the sequence $v_{n}$ is bounded, and has a subsequence, again $v_{n}$, converging uniformly to the harmonic $v(x)$ on compact sets of $A^{\prime} \cap \Omega^{\prime}$. In particular, the sequence $u_{n}(x)$ of biharmonic functions converges uniformly to $u(x)=w(x)+v(x)$ on $\partial \Omega$ itself. Since $T\left(f_{n}, g_{n}\right)=\left(\left.u_{n}\right|_{\partial \Omega},\left.h_{n}\right|_{\partial \Omega}\right)$ with each component converging uniformly, it follows that $T\left(f_{n}, g_{n}\right)$ converges in norm.

5. The Vector Space. $V \cap W$. The objective now is to use the RieszSchauder Theorem [7] to establish that the operator equation $(I-T)(\phi, \phi)$ $=P_{\partial \Omega}\left(s-D_{\alpha} \circ L \circ P_{\alpha}(s)\right)$ has a solution in the space $\mathrm{C}(\partial \Omega) \times \mathrm{C}(\partial \Omega)$. Since $T$ is already compact, it of course remains to show that the kernal $K$ of $I-T$ is zero. But if $(\phi, \phi) \in K$, then with $\left(3^{\prime}\right)$ there is a biharmonic $p$ on $R$ given by

$$
p= \begin{cases}D(\phi, \phi) & \text { on } \Omega, \\ L \circ P_{\alpha} \circ D(\phi, \phi) & \text { on } A .\end{cases}
$$

Now the harmonic $\Delta p$ attains extreme values on $\partial \Omega$, in particular, at interior points of $R$, and of course is constant. Since $\left.\Delta p\right|_{\partial \Omega}=\psi$, it follows that $\phi$ is constant as well.

Hence, we borrow from Rodin-Sario [1] the space $X \subset \mathrm{C}(\partial \Omega)$ for which $\int_{\partial \Omega} \phi * d \omega=0$, where $\omega$ is the harmonic measure of $A \cap \Omega$, satisfying $\left.\omega\right|_{\partial \Omega}=0$ 
and $\left.\omega\right|_{\alpha}=1$.

So in terms of $V=C(\partial \Omega) \times X$, if $(\phi, \phi) \in K \cap V$, then $\phi=$ const. $=0$. Hence, we conclude from the defining (iv) that $p=D(\phi, 0) \cup L \circ P_{\alpha} \circ D(\phi, 0)$ is itself harmonic and again constant. It follows that in the space $V$, the kernal $K$ of $T$ is the one dimensional $(k, 0)$. Next, the domain space of $T$ is further restricted with using the biharmonic measure $\sigma$ of Sario [3]: that is $\Delta \sigma=\omega$ with $\sigma$ vanishing on the boundary $\alpha \cup \partial \Omega$ and $\omega$ vanishing on $\partial \Omega$.

Definition. In terms of the biharmonic measure $\sigma$ the space $W \subset \mathrm{C}(\partial \Omega)$ $\times \mathrm{C}(\partial \Omega)$ is $\left\{(\phi, \phi) ; \int_{\partial \Omega} \phi * d \omega+\phi * d \sigma=0\right\}$.

Proposition. (a) In the Banach space $V \cap W$, the kernal $K$ of $I-T$ is $(0,0)$. (b) The linear operator $T$ takes $V \cap W$ into $V \cap W$. (c) The singularity $s-L \circ P_{\alpha} s$ $\in V \cap W$.

Proof of (a). It has been shown that if $(\phi, \phi) \in K \cap V$, then $(\phi, \phi)=(k, 0)$. But $(k, 0)$ can belong to $W$ only if $k=0$, and (a) is established.

Proof of (b). The verification that $T: V \rightarrow V$ is just that of [1] with $V$ here in the role of $X$ there. That is, the second component of $T(\phi, \phi)$ is $\left.\Delta L \circ P_{\alpha} \circ D(\phi, \psi)\right|_{\partial \Omega}$, and with Green's theorem along with the condition $\omega=0$ on $\partial \Omega$, we can write

$$
\begin{aligned}
\int_{\partial \Omega} \Delta L \circ P_{\alpha} \circ D(\phi, \phi) * d \omega & =\int_{\alpha} \Delta L \circ P_{\alpha} \circ D(\phi, \phi) * d \omega-\omega * d \Delta L \circ P_{\alpha} \circ D(\phi, \phi) \\
& =\int_{\alpha} \Delta D(\phi, \phi) * d w \\
& =\int_{\partial \Omega} \Delta D(\phi, \phi) * d \omega+\int_{\alpha} \omega * d \Delta D(\phi, \phi)=0 .
\end{aligned}
$$

Of course the term $\int_{\alpha} * d \Delta L \circ P_{\alpha} \circ D(\phi, \phi)=0$ since $L$ satisfies the flux condition (ii), and the fact that $T: V \rightarrow V$ follows from the observation that for $\phi \in V$, $\int_{\partial \Omega} \Delta D(\phi, \phi) * d w=\int_{\partial \Omega} \psi * d \omega=0$

To show that $W$ is invariant under $T$, we must consider the components $L \circ P_{\alpha} \circ D(\phi, \phi)$ and $\Delta L \circ P_{\alpha} \circ D(\phi, \phi)$ of $T(\phi, \phi)$ for $(\phi, \phi)$ in $W$. By means of an application of Green's formula (1) with the region $D$ as $\Omega \cap A$ and boundary $\partial D$ as $\partial \Omega \cup \alpha$, we obtain

$$
\begin{aligned}
\int_{\partial \Omega} L \circ P_{\alpha} \circ D(\phi, \phi) * d \omega+\Delta L \circ P_{\alpha} \circ D(\phi, \phi) * d \sigma \\
=\int_{\alpha} D(\phi, \phi) * d \omega+\Delta D(\phi, \phi) * d \sigma-* d L \circ P_{\alpha} \circ D(\phi, \phi)
\end{aligned}
$$




$$
\begin{aligned}
& =\int_{\partial \Omega} \phi * d \omega+\phi * d \sigma+\int_{\alpha} * d D(\phi, \phi)-* d L \circ P_{\alpha} \circ D(\phi, \phi) \\
& =\int_{\alpha} * d D(\phi, \phi)-* d L \circ P_{\alpha} \circ D(\phi, \phi) .
\end{aligned}
$$

However, from the defining (i) and (ii) for $L$, it follows that $\int_{\alpha} * d L \circ P_{\alpha} \circ D(\phi, \phi)$ $=\int_{\alpha} \Delta L * d v_{\alpha}=\int_{\alpha} \Delta D(\phi, \phi) * d v_{\alpha}$. On the other hand, in the region $\Omega_{\alpha}$ we have already applied Green's theorem with the biharmonic functions taken as $u=D(\phi, \phi)$ and $v=v_{\alpha}$ to see that $\int_{\alpha} * d D(\phi, \phi)=\int_{\alpha} \Delta D(\phi, \phi) * d v_{\alpha}$. Since this means $\int_{\alpha} * d D-* d L \circ P_{\alpha} \circ D=0$, it follows that $T(\phi, \phi) \in W$, and (b) is established.

Proof of (c). To see that $s-L \circ P_{\alpha}(s)$ belongs to $V \cap W$, it will be sufficient to apply Green's theorem again to the appropriate components, namely $s-\left.L \circ P_{\alpha} s\right|_{\partial \Omega}$ and $\Delta s-\left.\Delta L \circ P_{\alpha} s\right|_{\partial \Omega}$. With first using Green's formula for harmonic functions, we obtain

$$
\begin{aligned}
\int_{\partial \Omega}\left(\Delta s-\Delta L \circ P_{\alpha}(s)\right) * d \omega= & \int_{\partial \Omega} \omega * d\left(\Delta s-\Delta L \circ P_{\alpha}(s)\right)+\int_{\alpha}\left(\Delta s-\Delta L \circ P_{\alpha}(s)\right) * d \omega \\
& -\int_{\alpha} \omega * d\left(\Delta s-\Delta L \circ P_{\alpha}(s)\right)=0 .
\end{aligned}
$$

Hence it follows that $s-L \circ P_{\alpha}(s) \in V$.

Similar considerations along with another application of (1) are next used to show that $s-L \circ P_{\alpha}(s)$ belongs to $W$ as well. The integral or the appropriate components is

$$
\int_{\partial \Omega}\left(s-L \circ P_{\alpha}(s)\right) * d \omega+\Delta\left(s-L \circ P_{\alpha}(s)\right) * d \sigma=-\int_{\alpha} * d\left(s-L \circ P_{\alpha}(s)\right) .
$$

But, with the definition, each singularity s satisfies $\int_{\alpha} * d s=\int_{\alpha} \Delta s * d v_{\alpha}$, and on the other hand, due to the defining (i) and (ii) for the operator $L$, it follows that $\int_{\alpha} * d\left(L \circ P_{\alpha}(s)\right)=\int_{\alpha} \Delta L \circ P_{\alpha}(s) * d v_{\alpha}=\int_{\alpha} \Delta s * d v_{\alpha}$. Hence we have that $\int_{\alpha} * d\left(s-L \circ P_{\alpha}(s)\right)=0$ and it follows that $s-L \circ P_{\alpha}(s)$ does belong to $W$. With this, the proof of (c) is complete, as is the proof of the proposition.

Now, by virtue of the Riesz-Shauder theory [7], we are assured of a solution of the functional equation (3) in $V \cap W$, and with this solution, we may construct, from $\left(3^{\prime}\right)$, the biharmonic $p(x)$ for which $p-s$ is a regular 
biharmonic singularity; that is, $p-s=L \circ P_{\alpha}(p-s)$ on $A$. The theorem is now established with demonstrating the uniqueness.

6. Uniqueness. If $p_{1}$ and $p_{2}$ are biharmonic and satisfy (2), then of course $q=p_{1}-p_{2}$ is defined on $R$ and satisfies $q=L \circ P_{\alpha}(q)$ on $A$. So $\sup _{A}|\Delta q|=$ $\sup _{A}\left|\Delta L \circ P_{\alpha}(q)\right| \leqq\|\Delta q\|_{\alpha}$ holds for the harmonic $\Delta q$ which then has an interior point at which its maximum is attained. Hence, $\Delta q$ is constant, and with the hypothesis, equal to 0 . Now, with the defining properties (iii) and (iv), the same may now be said for the harmonic $p$, and this means, of course that $p_{1}=p_{2} \cdot$

7. A Principal Example. As an analogy with the $L_{1}$ operator of harmonic theory [Rodin-Sario ; 2], we shall construct here a biharmonic normal operator $L_{11}$. The manifold $R$ is taken as the interior of a subregion of a manifold $R^{\prime}$ containing $\bar{R}$ wherein the border is called $\beta$. Then the boundary neighborhood $A$ is $R-\Omega_{\alpha}$, where $\Omega_{\alpha}$ is a regular subregion with $\bar{\Omega}_{\alpha} \subset R$. And now, $L_{11}(f, g)$ is to be the biharmonic function for which $P_{\alpha} \circ L_{11}(f, g)$ as usual, and $P_{\beta} \circ L_{11}$ $=(k, l)$, where these constants are adjusted to meet the flux requirements (ii).

The construction is in part reminiscent of harmonic theory [4]. Because, in terms of $u$, the solution to the biharmonic Dirichlet problem in $A$, with $P_{\alpha}(u)=(f, g)$ and $P_{\beta}(u)=(0,0)$, we take $v$ as $u-\tau\left(\int_{\beta} * d \Delta u / \int_{\beta} * d \Delta \tau\right)$ where $\tau$ is a biharmonic measure on $A$ [Sario; 3]; that is, $\tau$ is 0 on $\alpha \cup \beta$, with Laplacian equal to 0 on $\alpha$ and 1 on $\beta$. Certainly $v$ now satisfies the condition $\int_{\alpha} * d \Delta v=0$, while $\Delta v$ is constant, say $l$, on $\beta$. It follows, from the maximum principle that $|l| \leqq\|g\|$. Next, $w$ is taken as hibarmonic on $A$, with $P_{\alpha}(w)=P_{\alpha}(v)=(f, g)$ and $P_{\beta}(w)=(k, l)$. Here, $k$ is to be selected in terms of integrals of the functions $f$ and $g$, the constant $l$ already chosen and the biharmonic measure $\sigma$ on $A$ for which $\Delta \sigma=\omega$ with $\omega=1$ on $\alpha$ and 0 on $\beta$. That is, since $\int_{\beta} * d \omega \neq 0, k$ may be chosen to satisfy

$$
\int_{\alpha} f * d \omega-g * d v_{\alpha}+g * d \sigma=\int_{\beta} k * d \omega+l * d \sigma .
$$

But according to Green's formula, the companion relation for the biharmonic $w$

$$
\int_{\alpha} f * d w-* d w+g * d \sigma=\int_{\beta} k * d \omega+l * d \sigma
$$

is valid. And now with a comparison of (6) and $\left(6^{\prime}\right)$ we observe that $\int_{\alpha} \Delta w * d v_{\alpha}=\int_{\alpha} g * d v_{\alpha}=\int_{\alpha} * d w$. Of course $L_{11}(f, g)$ is then taken as $w$, and the defining conditions (i) and (ii) are certainly satisfied. 
Furthermore, when $g$ is 0 , we would have that $w$ is harmonic for any choice of $k$. But the choice dictated by the comparison of (6) and $\left(6^{\prime}\right)$ is then $\int_{\alpha} f * d \omega=\int_{\beta} k * d \omega$. (This of course is the same old familiar condition that is needed to assure that the harmonic $w$ have zero flux.) Trivially, if $f=1$ identically, then $k=1$, and the defining (iv) is verified.

Hence there remains only the verification of (iii), the second part of which is similar to the harmonic theory [Rodin-Sario; 1 ]. In fact, since $\int_{\beta} * d \Delta L_{11}=0$, it follows that $L_{11}$ cannot be an extreme value on $\beta$, and so $\sup _{A}\left|\Delta L_{11}(f, g)\right|$ $\leqq\|g\|$.

For the purpose of studying $\sup _{A}|w(x)|$, as in section 4, we represent $w(x)$ in terms of the harmonic Green's function $g_{A}(x, y)$ as

$$
w(x)=\int_{A} g_{A}(x, y) * \Delta w(y)+h(x)
$$

where $h(x)$ is harmonic and has the data of $w(x)$ on $\alpha \cup \beta$. Hence the integral part of $(7)$ can be estimated as $|I(x)| \leqq \mu_{A}(\|g\| \vee l)=\mu_{A}\|g\|$, where $\mu_{A}=\sup _{A}(-\mathrm{G} 1(x))$. Since $\sup _{A}|h(x)| \leqq\|f\| \vee|k|$, an estimate for $w(x)$ is obtained from (6) which defines $k$; that is

$$
\begin{aligned}
& |k| \leqq\left(\int_{\beta} * d w\right)^{-1}\left[\int_{\alpha}\|f\| * d \omega+\|g\|\left(* d v_{\alpha}+* d \sigma\right)+\|g\| \int_{\beta} * d \sigma\right] . \\
& =\|f\|+b\|g\|
\end{aligned}
$$

The result is $|w(x)| \leqq \mu_{A}\|g\|+\|f\|+b\|g\|=\|f\|+\lambda\|g\|$ and the defining property (iii) is verified.

8. The Biharmonic Green's Function $\gamma$. Certainly now it is natural to attempt the construction of the Green's function $\gamma$ by means of an application of the $L_{11}$ operator. Unfortunately, such a construction using $L_{11}$ as one component in a direct sum of operators is not possible in the present stage of this theory. Nevertheless, the Green's function can be realized from the construction of an operator $L_{\gamma}$, and during the course of this construction, it will be easy enough to observe the reason for the failure of the present version of this theory to have the usual direct sum property.

Suppose that $R$ is a regular submanifold of the manifold $R^{\prime}$ and for $a \in R$, choose $V_{a}$ a parametic ball such that $V_{a} \subset \bar{V}_{a} \subset R \subset \bar{R} \subset R^{\prime}$. In terms of the coordinate $x$ in $V_{a}, q_{V_{a}}(x)$ is to denote the fundamental biharmonic singularity which satisfies $P_{\partial \Omega_{a}}\left(q_{V_{a}}\right)=(0,0)$ and whose Laplacian has flux +1 . The Green's function $\gamma_{R}(x, a)$ is then to have the singular behavior of $q_{V_{a}}(x)$ near $a$, and in terms of $\beta=\partial R$, is to satisfy $P_{\beta}\left(\gamma_{R}\right)=(0,0)$.

Of course the main theorem must be applied in a setting of a punctured manifold $R_{a}=R-a$, wherein a boundary neighborhood $N_{\beta}$ in $R$ is formed by first taking the regular subregion $\Omega_{0}$ for which $\bar{V}_{a} \subset \Omega_{0} \subset \bar{\Omega}_{0} \subset R$, and then 
forming $N_{\beta}=R-\bar{\Omega}_{0}$. In the punctured $R_{a}$, the regular region $\Omega_{\alpha}$ is taken as $\Omega_{0}-\bar{V}_{a}$ and the boundary neighborhood in $R_{a}$ is then $A=R_{a}-\bar{\Omega}_{\alpha}$. Now in $R_{a}, A=\left(V_{a}-a\right) \cup N_{\beta}$ and has boundary $\partial A=-\partial \Omega_{\alpha}=\alpha=-\left(\alpha_{\imath}+\alpha_{0}\right)$, where $\alpha_{\imath}=-\partial V_{a}$ and $\alpha_{0}=\partial \Omega_{0}$. In these terms, it is consistent to call $v_{\alpha}, v_{\alpha_{2}}$ and $v_{\alpha_{0}}$ those functions which are quasiharmonic on $\Omega_{\alpha}, V_{\alpha}$, and $\Omega_{0}$ and 0 on the boundaries $\alpha, \alpha_{\imath}$, and $\alpha_{0}$ respectively.

We call the operator $L$ from which the Green's function is to be realized by $L_{\gamma}(f, g)=w$ with $\left.w\right|_{N_{\beta}}=w_{0}$ and $\left.w\right|_{V_{a}}=w_{\imath}$ for $L_{\gamma}$ inside and outside respectively. First, $w_{\imath}$ is taken as the solution of the biharmonic Dirichlet problem in $V_{a}$ with $P_{\alpha_{i}}\left(w_{\imath}\right)=\left(f_{\imath}, g_{\imath}\right)$. Next, $w_{0}$ is to be biharmonic with $P_{\alpha_{0}}\left(w_{0}\right)=\left(f_{0}, g_{0}\right)$ and $P_{\beta}\left(w_{0}\right)=(k, l)$. The choice of the constant $l$ for which the flux $\int_{\beta} * d \Delta w=0$ is made again as it was for the $L_{11}$ operator. On the other hand, the constant $k$ must be chosen to satisfy a somewhat different matching equation

$$
\int_{\alpha_{0}} f_{0} * d \omega+g_{0} * d \sigma-g_{0} * d v_{\alpha}+\int_{\alpha_{\imath}} g_{i}\left(* d v_{\alpha_{\imath}}-* d v_{\alpha}\right)=\int_{\beta} k * d w+l * d \sigma .
$$

Such a $k$ depending on $f_{0}, g_{0}, f_{2}$ and $g_{\imath}$ can certainly be chosen because $\int_{\beta} * d \omega \neq 0$. With $k$ so selected, the biharmonic $w_{0}$ then satisfies the companion equation

$$
\int_{\alpha_{0}} w_{0} * d \omega+\Delta w_{0} * d \sigma-* d w_{0}=\int_{\beta} k * d \omega+l * d \sigma .
$$

Since $\int_{\alpha_{\imath}} * d w_{\imath}=\int_{\alpha_{\imath}} g_{i} * d v_{\alpha_{i}}$, a comparison of (9) and $\left(9^{\prime}\right)$ yields

$$
\int_{\alpha_{0}} * d w_{0}=\int_{\alpha_{0}} g_{0} * d v_{\alpha}-\int_{\alpha_{\imath}} * d w_{\imath}-g_{i} * d v_{\alpha}
$$

and this of course is just the flux requirement $\int_{\alpha} * d L_{\gamma}=\int_{\alpha} \Delta L_{\gamma} * d v_{\alpha}$. Hence the operator $L_{\gamma}(f, g)$ satisfies the defining (ii).

With a comparison of (6) for defining $\left.L_{11}(f, g)\right|_{\beta}$ with (9) for defining $\left.L_{\gamma}(f, g)\right|_{\beta}$, we see directly that (iii) for $L_{\gamma}$ is verified in just the same manner as it was for $L_{11}$. And the same can be said for the condition (iv).

To complete the construction of $\gamma(x, a)$, we need only select an appropriate biharmonic singularity $s(x)$. To start, $s(x)$ is taken as the fundamental negative biharmonic singularity $q_{V_{a}}(x)$ which satisfies $P_{\partial V_{a}}\left(q_{V_{a}}\right)=(0,0)$ and has flux $\int_{\partial V_{a}} * d \Delta q_{V_{a}}=+1$. Then in $N_{\beta}, s(x)$ is taken with $P_{\alpha_{0}}(s)=(0,0)$ and $P_{\beta}(s)=(m, n)$. The constants again taken so that the flux conditions are satisfied. That is, $\Delta s=t$, where $t=n(1-\omega)$ and $n=(-1) / \int_{\beta} * d \omega$. In terms of the orientation 
induced on $\alpha$ by $\Omega_{\alpha}$, we have $\int_{\alpha} * d \Delta s=0$. As usual, $m$ is then taken to satisfy the equation

$$
-\int_{\alpha_{\imath}} * d q_{V_{a}}=\int_{\beta} m * d \omega+n * d \sigma .
$$

With $m$ so defined, and $s(x)$ taken in $N_{\beta}$ as 0 on $\alpha$ and $m$ on $\beta$, the so-called companion equation for $s(x)$ is

$$
\int_{\alpha_{0}} * d s=\int_{\beta} s * d \omega+\Delta s * d \sigma
$$

and from comparing these, we have that $\int_{\alpha} * d s=0$. Since $\int_{\alpha} \Delta s * d v_{\alpha}=0$ as well, it follows that $\int_{\alpha} * d s=\int_{\alpha} \Delta s * d v_{\alpha}$ and the flux conditions for the singularity $s(x)$ are satisfied.

Finally, by virtue of an application of the Main Theorem with the operator $L$ and system of biharmonic singularities as just described, there exists the biharmonic $p=s+L_{\gamma} \circ P_{\alpha}(p-s)$, where $P_{\beta}(p)=\left(\kappa, \kappa^{\prime}\right)$, a pair of constant. In terms of $u$ for which $\Delta u=\kappa^{\prime}$ on $R$ and $\left.u\right|_{\beta}=\kappa$, the Green's function $\gamma$ is $p-u$.

\section{REFERENCES}

[1] Rodin, B. ANd L. SARio, Convergence of normal operators, Kōdai Math. Sem. Rep., 19 (1967), 165-173.

[2] Rodin, B. And L. SARIo, Principal functions, University Series, Van Nostrand, Princeton, 1968, pp. 347.

[3] Sario, L., Biharmonic measure, Ann. Acad. Sci. Fenn. A. I., 287 (1974).

[4] SARIO, L., A linear operator method on arbitrary Riemann surfaces, Trans. Amer. Math. Soc., 72 (1952), 281-295.

[5] SArio, L., M. NAKal, C. WANG, L. Chung, Classification theory of Riemannian manifolds, Lecture Notes in mathematics, 605, Springer Verlag, Berlin, Heidelberg, New York, 1977, pp. 498.

[6] Sario, L., M. Schiffer, M. Glasner, The span and principal functions in Riemannian spaces, J. Analyse Math., 15 (1965), 115-134.

[7] Yosida, K., Functional analysis, 2nd edition, Grundlehren 123, Springer Verlag, Berlin, Heidelberg, New York, 1968, pp. 465.

\section{North Carolina State University}

